\title{
Shuttle charging by fixed energy beam emissions
}

\author{
W. J. Burke, ${ }^{1}$ W. J. Raitt, ${ }^{2}$ D. C. Thompson, ${ }^{2}$ J. S. Machuzak, ${ }^{1}$ \\ L. C. Gentile, ${ }^{3}$ B. E. Gilchrist ${ }^{4}$ C. Y. Huang, ${ }^{3}$ D. L. Cooke, ${ }^{1}$ \\ D. A. Hardy, ${ }^{1}$ D. G. Olson, ${ }^{1}$ J.-P. Lebreton, ${ }^{5}$ and C. Bonifazi ${ }^{6}$
}

\begin{abstract}
We present environmental responses observed by the Shuttle Potential and Return Electron Experiment during 13 prolonged electron beam emissions from the Fast Pulsed Electron Generator (FPEG) during the TSS 1R deployment. As the tether lengthened from 0.18 to $2.5 \mathrm{~km}$, FPEG fired nine times at a nominal current of $100 \mathrm{~mA}$ at $1 \mathrm{keV}$. The motional potential induced across the system increased from 24 to $225 \mathrm{~V}$. With a $15 \Omega$ resistor connecting the tether to shuttle ground, current was higher and the degree of negative shuttle charging lower when FPEG was off than when it was on. While most FPEG firings left the shuttle uncharged, two created significant potentials: one to $+70 \mathrm{~V}$ in a nighttime, equatorial plasma depletion and one to $-60 \mathrm{~V}$, with the tether $\sim 15 \mathrm{~km}$.
\end{abstract}

\section{Introduction}

During the Tethered Satellite System reflight (TSS 1R) a conducting satellite was deployed above the shuttle Columbia by an electrically conducting tether. Orbital motion across the Earth's magnetic field generated an electromotive force to drive currents [Dobrowolny and Stone, 1994]. Current flow between the shuttle and local plasma was achieved through passive ion collection at conducting shuttle surfaces [Agüero et al., 1997; Gentile et al., 1997] or by electron beam emissions by two independent instruments. The first was the electron generator assembly (EGA), a diode emitter whose cathode was connected to the tether and anode to shuttle ground [Bonifazi et al., 1994]. The EGA operated over a wide range of beam currents, $I$, and energies specified by $I=6.4 \times 10^{-4} V^{\frac{3}{2}}$, where $V$ is the voltage between

\footnotetext{
${ }^{1}$ Phillips Laboratory, Hanscom AFB, Massachusetts.

${ }^{2}$ Center for Atmospheric and Space Science, Utah State University, Logan, Utah.

${ }^{3}$ Boston College Institute for Scientific Research, Chestnut Hill, Massachusetts.

${ }^{4}$ University of Michigan, Space Physics Research Laboratory, Ann Arbor, Michigan.

${ }^{5}$ Space Sciences Department, ESA-ESTEC, 2200 AG Noordwijk, The Netherlands.

${ }^{6}$ Agenzia Spaziale Italiana, Viale Regina Margherita 202, 00198 Rome, Italy.
}

Copyright 1998 by the American Geophysical Union.

Paper number 97GL03190.

0094-8534/98/97GL-03190\$05.00 the tether end and shuttle ground. The second, the Fast Pulsed Electron Generator (FPEG), emitted electrons at a fixed energy and current of $1 \mathrm{keV}$ and $100 \mathrm{~mA}$.

This paper presents ionospheric plasma responses to extended FPEG emissions detected in the payload bay by the Shuttle Potential and Return Electron Experiment (SPREE). We briefly describe SPREE, FPEG and the TSS 1R circuit during FPEG operations, then present SPREE spectral data for 13 FPEG firings. Two events illustrate positive and negative shuttle charging. The last section discusses the shuttle's electrical coupling to the ionosphere during FPEG emissions.

\section{Instrumentation and the TSS 1R Circuit}

The TSS payload is described in a special issue of Il Nuovo Cimento [1994]. We use measurements of: (1) electron and ion fluxes by SPREE [Oberhardt et al., 1994], (2) tether current, $I_{T}$, by the satellite ammeter [Bonifazi et al., 1994], (3) potentials between the tether and shuttle by a voltmeter in the payload bay [Agüero et al., 1994], and (4) electron densities, $n_{e}$, by a Langmuir probe (LP) on the satellite [Dobrowolny et al., 1994].

SPREE has two triquadraspherical electrostatic analyzers mounted on rotary tables. Electrons and ions from $9.8 \mathrm{eV}-10 \mathrm{keV}$ were sampled in 32 logarithmically spaced steps. Particles entered the apertures through angular fans $\sim 100^{\circ}$ by $8.5^{\circ}$ in 10 zones looking from shuttle-horizontal to shuttle-zenith. Full energy spectra were compiled simultaneously at rates of 1 or $8 \mathbf{s}^{-1}$. The full $2 \pi$ sr upper hemisphere was sampled in $30 \mathrm{~s}$.

FPEG was designed to emit a nominal current of 100 $\mathrm{mA}$ in dc or pulsed $(\leq 30 \mathrm{kHz})$ modes. Electrons were ejected with an energy of $1 \mathrm{keV}$ through a $1 \mathrm{~cm}^{2}$ aperture aimed $23^{\circ}$ above the shuttle's right wing. In sequence, one of four resistors, $R_{S}$, of $2.5 \mathrm{M} \Omega, 250 \mathrm{k} \Omega, 25$ $\mathrm{k} \Omega$, or $15 \Omega$, could be inserted between the tether and shuttle ground [Agüero et al., 1994].

Figure 1 represents the TSS 1R circuit for FPEG operations. Columbia flew engine bells-to-ram to facilitate ion current collection. The satellite, deployed upward to a distance, $L$, on the high potential end of the tether, collected ionospheric electrons. The circuit included a voltmeter and two switches. With $S_{1}$ open and FPEG off, no current flowed and no sheaths formed about the shuttle or satellite. Thus, $V_{1,2}$ was a measure of the induced potential $\Phi_{0}=\left(-V_{S} \times \mathbf{B}\right) \cdot \mathbf{L}$, where $\mathbf{V}_{S}$ and $\mathbf{B}$ are shuttle velocity and magnetic field. With $S_{1}$ closed, 


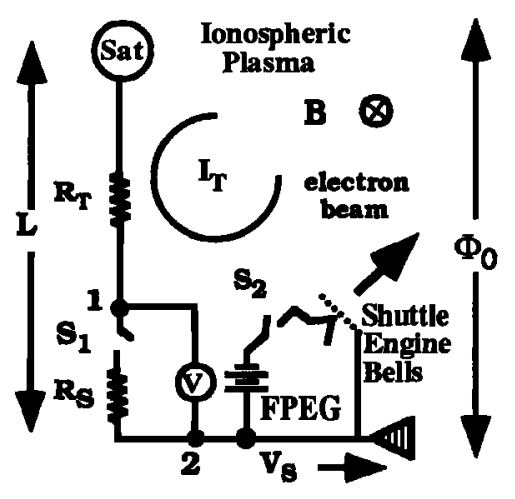

Figure 1. TSS 1R circuit during FPEG operations.

$V_{1,2}=R_{S} I_{T}$. FPEG operated with $S_{1}$ either open or closed. SPREE data were taken with both $S_{1}$ and $S_{2}$ closed and $R_{S}=15 \Omega$ or $25 \mathrm{k} \Omega$. Gentile et al. [1997] describe cases with the same resistors and FPEG off.

\section{Observations}

The first experiment occurred early in the deployment. As shown in the top plot of Figure 2, it had 4 circuit configurations, each 100 s. (1) The circuit was passive $\left(S_{1}\right.$ and $S_{2}$ open) and $V_{1,2}=\Phi_{0}$. (2) FPEG turned on, ( $S_{1}$ open, $S_{2}$ closed). (3) FPEG continued firing and resistors were inserted from high to low impedance into the circuit ( $S_{1}, S_{2}$ closed). (4) FPEG turned off and resistors were reinserted in sequence ( $S_{1}$ closed, $S_{2}$



Figure 2. TSS 1R data for 2225 - 2233 UT on February 25, 1996. Plot a gives tether length, time of FPEG firings, and resistor insertions. Plots $b$ and $c$ show voltmeter and ammeter readings, respectively. Plot $d$ contains shuttle potentials inferred from SPREE spectra. Plot e indicates electron density. open). Table 1 summarizes firings with the $15 \Omega$ resistor. The columns list: (1) event number, (2) universal time, UT, of resistor insertion on February 25, 1996, (3) tether length, $L,(\mathrm{~km}),(4)$ open-circuit potential, $\Phi_{0}$, (volts), (5) tether current, $I_{T},(\mathrm{~mA})$ with FPEG on, (6) tether current, $I_{T}^{\prime}$, measured $\sim 1$ min later with $R_{S}=$ $15 \Omega$ but FPEG off, (7) shuttle local time, $L T$, and (8) electron density, $n_{e}\left(\mathrm{~cm}^{-3}\right)$.

Data in Table 1 and SPREE spectra (not shown) support several conclusions: (1) SPREE detected electron fluxes $>10^{11} \mathrm{~cm}^{-2} \mathrm{~s}^{-1}$ throughout the first FPEG emission. Spectra decreased monotonically with energy to several hundred $\mathrm{eV}$. No ion fluxes were detected, indicating low levels of shuttle charging. (2) SPREE detected no fluxes above photoelectron background during events 3-8. Similar appearance/disappearance cycles for superthermal electrons occurred during FPEG firings after the tether break and are not tether related. Trajectory anaysis shows that beam electrons impacted insulating shuttle surfaces at these times. (3) Ion fluxes, detected only in event 8 , indicate that the shuttle charged to $-15 \mathrm{~V}$. (4) The shuttle charged positively during event 9 as it passed through an equatorial plasma depletion. (5) With $R_{S}=15 \Omega, I_{T}>I_{T}^{\prime}$ and the shuttle charged more negatively when FPEG was off than when it was on (Gentile et al. [1997]).

Plate $1 \mathrm{~A}$ is an energy-vs-time color spectrogram of directional differential fluxes $\left(\mathrm{cm}^{-2} \mathrm{~s}^{-1} \mathrm{sr}^{-1} \mathrm{eV}^{-1}\right)$ of electrons (top) and ions (middle) reaching SPREE during event 9 . The bottom plot shows $R_{S}$ insertions. When FPEG turned on at 2227:36 UT, electron fluxes first rose then fell. From 2218:15 UT until FPEG turned off, intense electron fluxes were detected. Persistent peaks appear in electron spectra after 2228:30 UT showing that the shuttle charged positively with respect to the local plasma. The charging-peak energy rose to $>70$ $\mathrm{eV}$, fell to $32 \mathrm{eV}$, then stabilized near $50 \mathrm{eV}$.

Figure 2 is an overview of TSS 1R data for 2225 2233 UT. Figure 2a plots $L$, increasing from $\sim 2$ to 2.7 $\mathrm{km}$, and times of FPEG firing and resistor insertions. Figure 2b records voltmeter readings. With the $15 \Omega$ resistor inserted, the tether was essentially short circuited to shuttle ground and the voltmeter measured very low potentials. With the $25 \mathrm{k} \Omega$ resistor in place,

Table 1. FPEG Firing Events of Experiment 1

\begin{tabular}{|c|c|c|c|c|c|c|c|}
\hline No. & UT & L & $\Phi_{0}$ & $\mathbf{I}_{\boldsymbol{T}}$ & $I^{\prime} T$ & LT & $\mathrm{n}_{e}$ \\
\hline 1 & 2136:35 & 0.18 & 24 & 10 & 2 & $05: 18$ & $1.5 \times 10^{5}$ \\
\hline 2 & 2143:03 & 0.24 & 38 & 16 & 6 & 07:06 & $2.1 \times 10^{5}$ \\
\hline 3 & 2149:53 & 0.35 & 50 & 27 & 11 & $08: 30$ & $8.0 \times 10^{5}$ \\
\hline 4 & $2156: 25$ & 0.54 & 65 & 29 & 24 & $10: 30$ & $2.0 \times 10^{6}$ \\
\hline 5 & 2203:05 & 0.69 & 83 & 39 & 19 & $12: 12$ & $3.0 \times 10^{6}$ \\
\hline 6 & $2209: 47$ & 0.98 & 122 & 52 & 21 & $14: 00$ & $1.0 \times 10^{6}$ \\
\hline 7 & 2216:00 & 1.43 & 165 & 76 & 36 & $15: 42$ & $9.0 \times 10^{5}$ \\
\hline 8 & 2223:08 & 2.19 & 203 & 93 & 55 & $17: 42$ & $2.0 \times 10^{6}$ \\
\hline 9 & 2229:50 & 2.39 & 443 & 6 & 2 & $19: 30$ & $6.0 \times 10^{4}$ \\
\hline
\end{tabular}




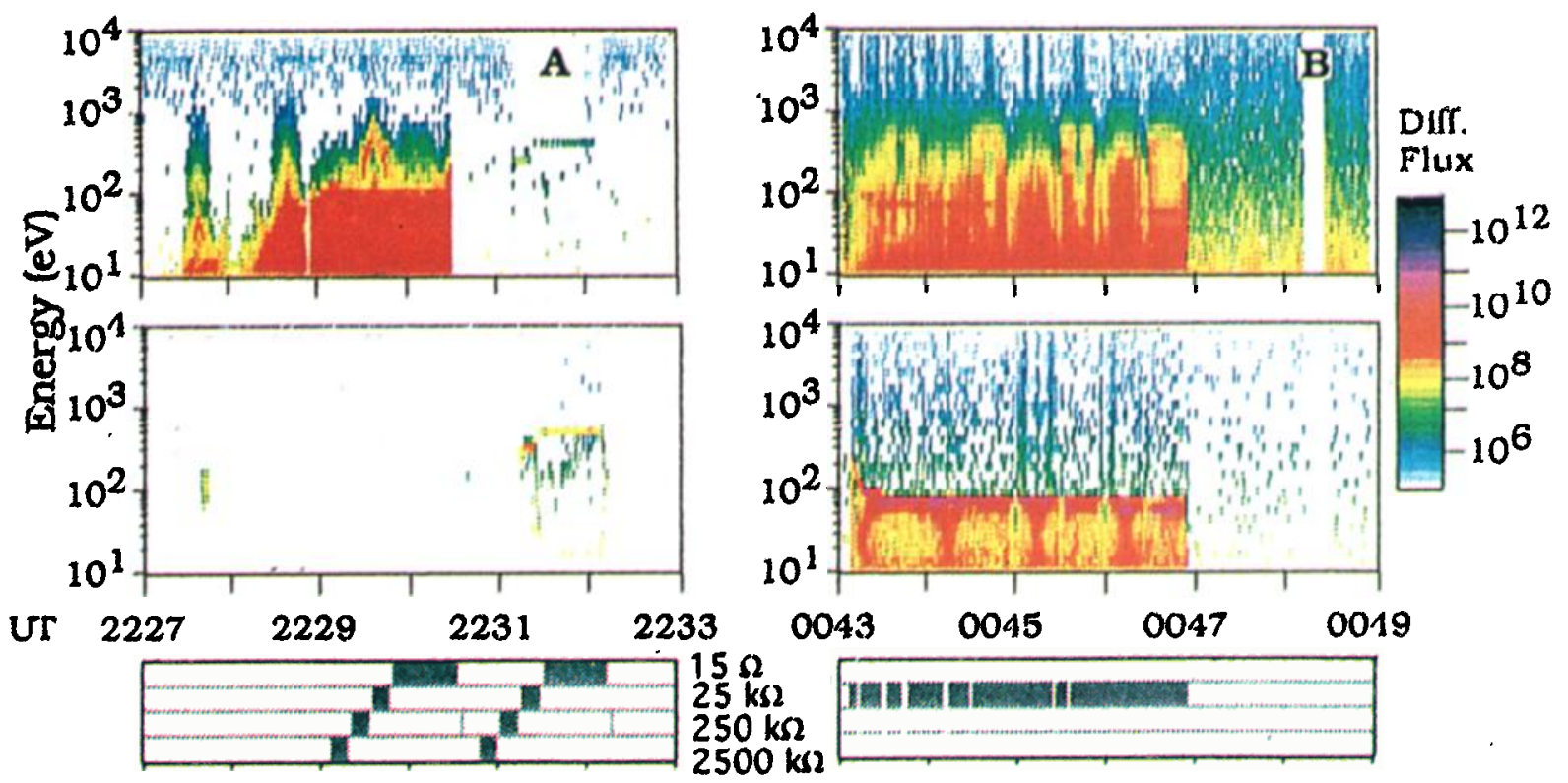

Plate 1. Energy-vs-time spectrograms of SPREE electron and ion fluxes (A) $2227-2233$ UT on February 25, 1996, and (B) 0043 - 0049 UT on February 26, 1996.

potentials fell from 90 to $83 \mathrm{~V}$ (after 2229:36 UT) and from 93 to $65 \mathrm{~V}$ (after 2231:18 UT). When the circuit was open or high-impedance resistors were in place, $V_{1,2}$ rose slowly from $\sim 200 \mathrm{~V}$ to $225 \mathrm{~V}$ at $2228: 30 \mathrm{UT}$, then rapidly to $325 \mathrm{~V}$. During this rapid transition, the magnetic field and shuttle attitude changed only slightly, thus $\Phi_{0}$ should have increased slowly. After the $15 \Omega$ resistor was removed and FPEG turned off, $V_{1,2}$ was nearly constant at $225 \mathrm{~V}$. Figure $2 \mathrm{c}$ shows a remarkable decrease in $I_{T}$ between the first (22:25 UT) and second/third $15 \Omega$ resistor insertions. Figure $2 \mathrm{~d}$ records shuttle potential variations inferred from SPREE data and Figure $2 e$ gives electron density at the satellite. As a whole the data show that the positive shuttle charging during the FPEG firing was not strongly dependent on whether any resistor was in the circuit but did coincide with: (1) a rapid density decrease from $\sim 10^{5} \mathrm{~cm}^{-3}$ to $<10^{4} \mathrm{~cm}^{-3}$ and (2) an increase in $V_{1,2}$ from 225 to 325 V. Finally, while $\Phi_{S}$ of $-90 \mathrm{~V}$ was sufficient to draw ion current of $\sim 60 \mathrm{~mA}$ with $n_{e} \approx 10^{6} \mathrm{~cm}^{-3}(2225 \mathrm{UT})$, $-245 \mathrm{~V}$ was required to draw $\sim 2 \mathrm{~mA}$ (Table 1) with $n_{e}$ $\approx 10^{4} \mathrm{~cm}^{-3}$ (2232 UT).

Experiment 2 included two 12 min sequences, each with a different resistor, which were run twice. For each sequence, the circuit was initially passive for $4 \mathrm{~min}\left(S_{1}\right.$, $S_{2}$ open), the EGA fired for 4 min [Burke et al., 1997], then FPEG fired for $4 \min \left(S_{1}, S_{2}\right.$ closed). $R_{S}$ was 25 $\mathrm{k} \Omega$ or $250 \mathrm{k} \Omega$ during the FPEG cycles. Data from the
FPEG firings are given in Table 2 in a format similar to that of Table 1. The last column lists $R_{S}$. SPREE detected intense electron fluxes during all 4 events. $I_{T}$ was nearly constant except during event 3 when it rose steadily from 95 to $120 \mathrm{~mA}$. No shuttle charging was evident with $R_{S}=250 \mathrm{k} \Omega$. In event 1, SPREE detected no ion fluxes and electron spectra peaked at $\sim 15 \mathrm{eV}$ indicating positive shuttle charging. The only strong negative shuttle charging occurred during the second $25 \mathrm{k} \Omega$ insertion near the dawn terminator. The LP was off as emission began, but measured a plasma density of $\sim 10^{6} \mathrm{~cm}^{-3}$ immediately after FPEG turned off. Plate 1B shows SPREE electron and ion fluxes for this event. A clear ion charging peak is seen at $\sim 60 \mathrm{eV}$ with periodic ion fluxes for all energies $10-60 \mathrm{eV}$ at $1 \mathrm{~min}$ intervals when SPREE looked toward FPEG. A similar effect was observed during EGA emission at 0051 UT when SPREE faced the EGA beam (Plate 1D of Burke et al., [1997]). Ions with intermediate energies were created within the sheath in collisions between beam electrons and neutrals.

\section{Summary and Discussion}

The data demonstrate that FPEG emissions did affect the TSS 1R circuit, and under different circumstances left the shuttle charged positively or negatively. More subtle effects were also present. With $R_{S}=15$

Table 2. FPEG Firing Events of Experiment 2

\begin{tabular}{cccrccrrc}
\hline No. & UT & $\mathrm{L}$ & \multicolumn{1}{c}{$\mathrm{R}_{\boldsymbol{T}}$} & \multicolumn{1}{c}{$\Phi_{0}$} & \multicolumn{1}{c}{$\Phi_{S}$} & $\mathrm{I}_{\boldsymbol{T}}$ & $\mathrm{LT}$ & $\mathrm{n}_{e}$ \\
\hline 1 & $2351: 55$ & 8.89 & $25 \mathrm{k} \Omega$ & 1547 & +15 & 55 & $17: 18$ & $1.2 \times 10^{6}$ \\
2 & $0003: 55$ & 9.93 & $250 \mathrm{k} \Omega$ & 1223 & & 2 & $20: 06$ & $5.0 \times 10^{5}$ \\
3 & $0043: 05$ & 14.6 & $25 \mathrm{k} \Omega$ & 3060 & -60 & $95-120$ & $06: 35$ & $1.0 \times 10^{6}$ \\
4 & $0055: 05$ & 16.2 & $250 \mathrm{k} \Omega$ & 3560 & & 10 & $09: 30$ & $1.5 \times 10^{6}$ \\
\hline
\end{tabular}


$\boldsymbol{\Omega}$, more current flowed through the tether. Also, the shuttle charged less negatively when FPEG was on than when it was off. These observations are related through requirements of Ohm's and Kirchoff's laws. Ohm's law determines how $\Phi_{0}$ is distributed between the sheath potentials of the satellite $\Phi_{S a}$ and shuttle $\Phi_{S}$ and resistive potential drops $I_{T}\left(R_{S}+R_{T}\right)$. Kirchoff's law demands current continuity at any node. Thus, if the satellite collects electron current $I_{T}$ and FPEG emits an electon current of $100 \mathrm{~mA}$, then an electron current $\left(100-I_{T}\right)$ must return to the shuttle from the ionosphere. Hardy et al. [1995] showed that FPEG emissions produce warm electron populations near the shuttle consisting of beam particles, collision-generated secondaries and heated ambient electrons. Return currents $\left(100-I_{T}\right)$ determine the level of shuttle charging. During experiment 1 the return current from the dayside ionosphere was such that $-15 \mathrm{~V}<\Phi_{S}<10 \mathrm{~V}$. Since $\Phi_{S}$ was more negative with $R_{S}=15 \Omega$ and FPEG off, a larger fraction of $\Phi_{0}$ was available for $\Phi_{S a}$ than when FPEG was on. The higher values of $\Phi_{S a}$ allowed the satellite to collect more ionospheric electrons [Stone and Bonifazi, 1997].

During event 9 , the satellite extracted only $6 \mathrm{~mA}$ of current from the low-density, nightside ionosphere. To provide an electron return current of $94 \mathrm{~mA}$ to the shuttle required that $\Phi_{S}$ reach $>70 \mathrm{~V}$. There remains an unresolved difference between the SPREE and voltmeter data. From Ohm's Law, $V_{1,2}=\Phi_{0}+\Phi_{s}$. Throughout this event the induced potential $\Phi_{0}$ should have remained nearly constant. Figure $2 \mathrm{~b}$ shows that although $V_{1,2}$ and $\Phi_{S}$ followed similar upward trends, SPREE spectra indicate that $\Phi_{S}$ rose by $\sim 70 \mathrm{~V}$, while the voltmeter reading increased $\sim 100 \mathrm{~V}$. A $30 \mathrm{eV}$ difference is within the energy-resolution capability of SPREE.

The shuttle charged negatively twice with FPEG emitting. (1) During event 8 of experiment $1, \Phi_{S}$ was $-15 \mathrm{~V}$, but no electron fluxes reached SPREE. $I_{T}$ remained steady at $93 \mathrm{~mA}$. If FPEG emitted at $100 \mathrm{~mA}$, there is a $7 \mathrm{~mA}$ deficit that must be made up of electrons with energies outside the sheath $>15 \mathrm{eV}$. The flux of these electrons to shuttle conducting surfaces must exceed the accelerated ion ram current by $7 \mathrm{~mA}$. (2) With $R_{S}=25 \mathrm{k} \Omega$ and FPEG emitting (event 3 of experiment 2), the shuttle charged to $-60 \mathrm{~V}$. During the 4 min firing $I_{T}$ systematically rose from 95 to $120 \mathrm{~mA}$. If FPEG emissions were nominal, the return current (100 - IT) changed from a $5 \mathrm{~mA}$ surplus of electrons with energies $>60 \mathrm{eV}$ to a $15 \mathrm{~mA}$ surplus of ions. The reason for this is unclear. Based on FPEG current drifts observed during preflight tests, it appears likely that the emitted current drifted from its nominal value so that the tether and FPEG currents were approximately equal. In this case, the measured value of $\Phi_{S}$ is the floating potential required to attract as many ions from the ionosphere as beam-heated electrons reached conducting shuttle surfaces.

Acknowledgments. This work was supported by the U. S. Air Force Office of Scientific Research task 2311PL014 and by Air Force contract F19628-96-K-0030 with Boston College. The authors thank N. Bonito, C. Roth, and E. Courtney of RADEX, Inc., who designed and maintained the SPREE software.

\section{References}

Agüero, V., P. M. Banks, B. Gilchrist, I. Linscott, W. J. Raitt, D. Thompson, V. Tolat, A. B. White, S. Williams, and P. R. Williamson, The shuttle electrodynamic tether system (SETS) on TSS 1, Nuovo Cimento, 17, 49-65, 1994.

Bonifazi, C., F. Svelto, and J. Sabbagh, TSS Core equipment, 1. Electrodynamic package and rationale for system electrodynamic analysis, Nuovo Cimento, 17, 13-47, 1994.

Burke, W. J., C. Bonifazi, D. A. Hardy, J. S. Machuzak, L. C. Gentile, D. G. Olson, C. Y. Huang, B. E. Gilchrist, J. -P. Lebreton, and C. A. Gurgiolo, Shuttle charging by tether controlled electron beam, Geophys. Res. Lett., this issue, 1997.

Dobrowolny, M., and N. H. Stone, A technical overview of TSS-1: The first tethered-satellite mission, Nuovo $\mathrm{C} i$ mento, 17, 1-12, 1994.

Dobrowolny, M., E. Melchioni, U. Guidoni, L. Iess, M. Maggi, R. Orfei, Y. de Conchy, C. C. Harvey, R. M. Manning, F. Wouters, J.-P. Lebreton, S. Ekholm, and A. Butler, The RETE experiment for the TSS-1 mission, Nuovo Cimento, 17, 101-121, 1994.

Gentile, L.C., W. J. Burke, J. S. Machurak, C. Y. Huang, D.A. Hardy, D. G. Olson, B. E. Gilchrist, C. Bonifazi, J. -P. Lebreton, Negative shuttle charging during TSS IR, Geophys. Res. Lett., Tethered Satellite Reflight, Part 1, 1997.

Hardy, D. A., M. R. Oberhardt, W. J. Burke, D. C. Thompson, W. J. Raitt, and L. C. Gentile, Observations of electron beam propagation perpendicular to the Earth's magnetic field during the TSS 1 mission, J. Geophys. Res., 100, 21523-21534, 1995.

Oberhardt, M. R., D. A. Hardy, W. E. Slutter, J. O. McGarity, D. J. Sperry, A. W. Everest III, A. C. Huber, J. A. Pantazis, and M. P. Gough, The shuttle potential and return electron experiment, Nuovo Cimento, 17, 67-83, 1994.

C. Bonifazi, Agenzia Spaziale Italiana, Viale Regina MaIgherita 202, 00198 Rome, Italy. e-mail: bonifazi@asirom. rm.asi.it

W. J. Burke, D. L. Cooke, D. A. Hardy, J. S. Machuzak, and D. G. Olson, Phillips Laboratory, 29 Randolph Road, Hanscom AFB, MA, 01731-3010. e-mail: burke@plh.af.mil; cooke@plh.af.mil; hardy@plh.af.mil; john.machuzak@psfc. mit.edu; olson@plh.af.mil

L. C. Gentile and C. Y. Huang, Boston College Institute for Scientific Research, 402 St. Clement's Hall, 140 Commonwealth Avenue, Chestnut Hill, MA 02167-3862. e-mail: gentile@plh.af.mil; huang@plh.af.mil

B. E. Gilchrist, University of Michigan, Space Physics Research Laboratory, 2455 Hayward Street, Ann Arbor, MI 48109-2143. e-mail: gilchrst@eecs.umich.edu

J.-P. Lebreton, ESA/ESTEC, Mail Code 50, Kepleriann 1, 2200 AG Noordwijk, The Netherlands. e-mail: jlebreto@ estec.esa.nl

W. J. Raitt and D. C. Thompson, Center for Atmospheric and Space Science, Utah State University, Logan, UT 84322-4405. email: raitt@cass.usu.edu; thompson@demise. cass.usu.edu

(Received January 13, 1997; revised October 3, 1997; accepted October 20, 1997.) 